\title{
Hardware in the loop verification of a fast backup protection scheme for embedded MVDC links within distribution networks
}

\author{
Lewis Hunter \\ Electronic and Electrical Engineering \\ University of Strathclyde \\ Glasgow, Scotland \\ lewis.hunter.100@strath.ac.uk
}

\author{
Qiteng Hong \\ Electronic and Electrical Engineering \\ University of Strathclyde \\ Glasgow, Scotland \\ q.hong@strath.ac.uk
}

\author{
Campbell Booth \\ Electronic and Electrical Engineering \\ University of Strathclyde \\ Glasgow, Scotland \\ campbell.d.booth@strath.ac.uk
}

\author{
Stephen Finney \\ Electronics and Electrical Engineering \\ The University of Edinburgh \\ Edinburgh, Scotland \\ stephen.finney@ed.ac.uk
}

\author{
Adrià Junyent-Ferré \\ Electrical and Electronic Engineering \\ Imperial College London \\ London, UK \\ adria.junyent-ferre@imperial.ac.uk
}

\begin{abstract}
With increasing interest in the deployment of dc technologies in ac distribution networks, there is a need to ensure compatibility with existing protection systems. This paper presents findings from hardware in the loop investigations which verify a novel approach to providing backup protection to an embedded dc link. This approach reconfigures existing digital distance protection devices to provide fast acting backup protection to embedded dc links whilst still being sensitive to conventional ac side zone faults. A brief overview of medium voltage dc trial projects in Great Britain is also provided.
\end{abstract}

Keywords-Distance Protection, Embedded Link, MVDC, Power System Protection, Smart Grid.

\section{INTRODUCTION}

There is growing interest amongst distribution network operators (DNO) in deploying medium voltage direct current (MVDC) technologies in their networks as they shift towards the future distribution system operator (DSO) model. Comparatively small levels of embedded MVDC, in the correct location, have been proven (both academically and via deployment trials [1] [2] [3] [4]) to provide increased control of existing assets. This can enable increases in distributed generation (DG) and future load which may otherwise require unaffordable, extensive and disruptive conventional network reinforcement. While MVDC is attracting interest both from network companies and equipment manufacturers, there are still technical integration considerations which need to be addressed and developed to increase customer confidence in the technology.

MVDC protection schemes need to be fast acting, especially for dc side faults, otherwise the power electronic devices are likely to be damaged [5]. This is true not just for main protection, but also for backup schemes. Previous studies have considered what a dc side fault may look like to conventional ac protection [6]. The role of a modified ac side distance protection scheme to provide sufficiently fast acting backup protection for embedded MVDC links appears promising under a desktop simulation environment; however has been unproven on real hardware.
This paper therefore aims to validate the use of an existing commercially available distance protection intelligent electronic device (IED) as a backup protection method in a real time, hardware in the loop, simulation environment. The paper also gives an overview of MVDC demonstration projects in Great Britain (GB).

\section{BACKGROUND}

Embedding MVDC within distribution networks requires detailed technical interfacing between the DNO and equipment providers. In particular the operational requirements, working environment, regulatory conditions, expected availability and maintenance requirements may be different from what equipment providers are used to providing for more mainstream medium power dc solutions. Converters of similar power ratings are more generally deployed as machine drives for marine, mining and industrial process applications.

When interfacing MVDC links within distribution networks, care needs to be exercised to ensure the stability of existing ac side protection. The following subsections will provide a brief overview of the conventional setting of ac side distance protection and the proposed fast backup method.

\section{A. Distance Protection Philosophy}

Distance protection relies upon measurement of both voltage and current at a given point on a network; Fig. 1. Using complex ohms law, the impedance, of the network, can be calculated for phase to phase and phase to ground during normal and faulted conditions [7].

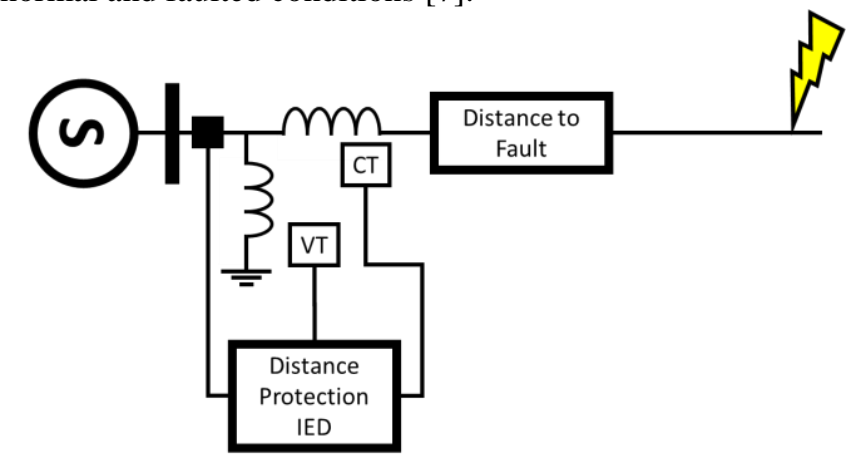

Fig. 1. Distance protection system connection topology 
Using the impedance measurement and the known parameters of the network (cable, line, load and transformer impedances) it is possible to compute the distance to a fault on the network should one arise [8]. From this, it is possible to create operational zones, typically but not limited to three, to be defined with tripping times specified for each zone to provide discrimination with downstream protection devices. This makes distance protection ideal for both main and backup protection purposes [9]. Zones can be of many geometric shapes which include lenticular, quadrilateral, circular and the offset Mho characteristic outlined in Fig. 2.

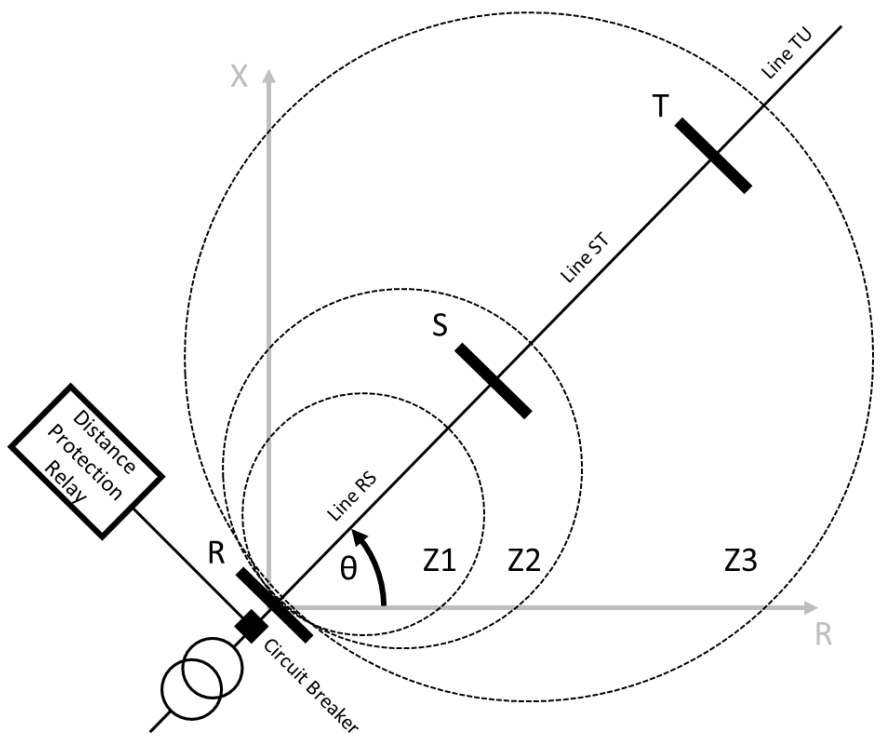

Fig. 2. Distance protection offset Mho reach

With reference to Fig. 2, zone 1 (Z1) protects $80 \%$ of the protected feeder (RS). This reduction in impedance is to ensure that $\mathrm{Z} 1$ does not over-reach and trip in the case of a fault on line ST near to bus-bar S. Over-reach may be caused by errors and variations in line parameters and transducer uncertainty. Zone 2 (Z2) is set to $120 \%$ of RS while Z3 typically reached to $120 \%$ of line ST. Table I presents maximum clearance times used by the Scottish DNO Scottish and Southern Energy Networks (SSEN) [10].

TABLE I

MAXIMUM CLEARANCE TIMES FOR DISTANCE PROTECTION SCHEMES

\begin{tabular}{cl}
\hline \hline Zone & Clearance time (s) \\
\hline $\mathrm{Z} 1$ & 0.150 \\
$\mathrm{Z} 2$ & 0.500 \\
$\mathrm{Z} 3$ & 1.300 \\
\hline \hline
\end{tabular}

\section{B. Proposed Protection strategy}

When a fault on an embedded dc link occurs, a large inductive impedance is observed from ac side protection. The inductive nature of this fault is due to the significant converter filter inductance used to smooth the converter's output. This value is equal to the sum of the impedances through which the current flows to the fault location; including ac network, converter arm, transformer, filter and dc line impedances [6]. The proposed protection strategy is to deploy a fast zone four trip signal when impedances are measured in the area identified in Fig. 3.

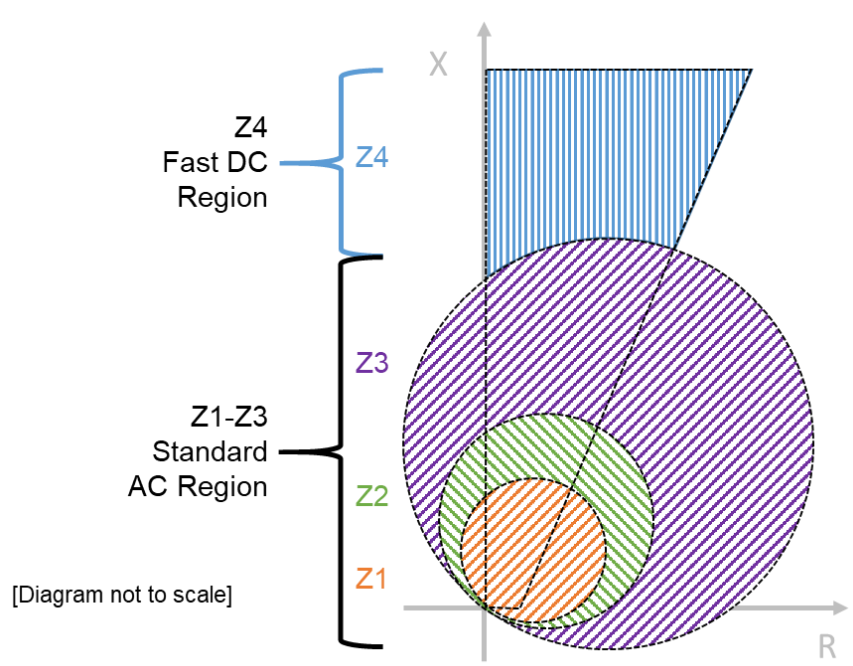

Fig. 3. Proposed distance protection relay configuration including accelerated zone four tripping region

The $\mathrm{Z} 4$ resistive and reactive reaches are approximated via Equation (1) and (2) where a grading margin of $20 \%$ is added to the calculated impedance to take into account an uncertainty in values. The use of a quadrilateral shape allows for large inductive reaches to be set without encroaching along the resistive $\mathrm{x}$-axis; this allows for greater configuration flexibility under high load and/or short line conditions when compared to the mho-type approach [9].

$$
\begin{gathered}
R_{Z 4} \approx 1.2 \times \sum\left[R_{\text {Line }_{a c}}, R_{\text {filter }}, R_{\text {Line }_{d c}}\right] \\
X_{Z 4} \approx 1.2 \times \sum\left[X_{\text {Line }_{a c}}, X_{\text {Reactor }}, X_{\text {Filter }}, X_{\text {Line }_{\text {dc }}}\right]
\end{gathered}
$$

\section{PRE EXPerimental (OfFline) Modelling}

\section{A. Network modelling}

The test network used for the following studies is outlined in Fig. 4. The network is based upon a section of existing Scottish distribution network where the introduction of controlled MVDC links appears promising. In this example two circuits are fed from bus-bar B. The first circuit is a $10 \mathrm{~km}$ ac line which supplies a $4 \mathrm{MW}$ load. The second circuit connects to an external grid via an MVDC link, via the repurposing of existing ac conductors, giving the local DSO greater operational flexibility of existing system assets. All conductors use the same characteristic impedance of $(0.17+j 0.3) \Omega / \mathrm{km}$ and are modelled as lumped element R-L series components.

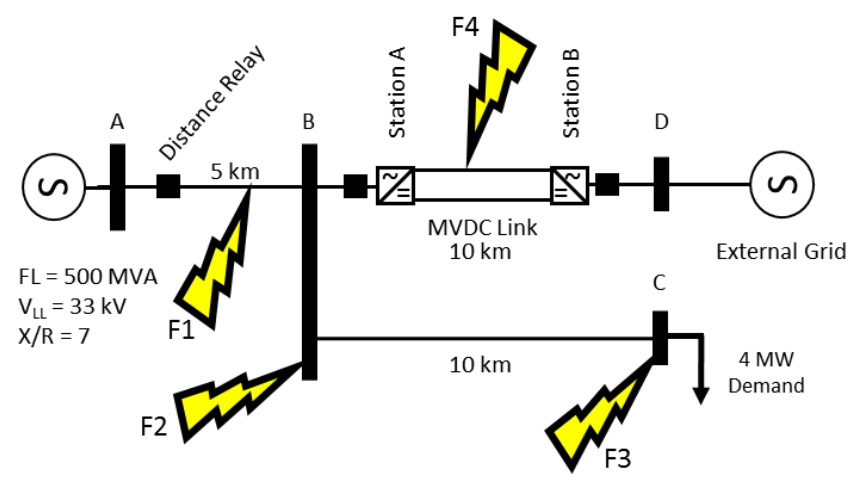

Fig. 4. Test Distribution Network Single Line Diagram with fault locations indicated 
The network was originally protected via a distance protection relay which is configured in line with the DSO's guidelines [10]. The aim of this paper is to determine whether the proposed strategy can identify faults on the MVDC link and distinguish them from a similarly placed fault on the parallel ac feeder. Fig. 4 also outlines four fault locations, one in each protection zone, which will be studied under the proposed test environment. For conciseness, faults are modelled as symmetrical phase to phase to earth for ac side disturbances and pole to pole to earth for dc side events.

\section{B. Converter modelling}

The converter used for these simulations is based upon a two level, symmetrical monopole, arrangement of 35 MVA rating with an operating dc voltage of $\pm 27 \mathrm{kV}$. The control for these converters is based on standard inverter control with one station operating under a P-Q mode while the other follows a $\mathrm{V}_{\mathrm{dc}}-\mathrm{Q}$ set point [11]. During ac side faults, it is assumed for these studies that the dc link provides grid support. There are, at present, no grid codes which govern the connection and fault ride through requirements of such a link to a distribution network in GB [12]. The converter has a per unit filter impedance of $20 \%(6.22 \Omega / 19.8 \mathrm{mH})$. This is in addition to an ac side reactor of $10 \mathrm{mH}(3.14 \Omega)$ introduced into the circuit to limit fault current to the MVDC link.

During dc faults the converter should block commutation of switching devices and open ac side converter circuit breakers at both converter stations. This should occur within five ac cycles $(100 \mathrm{~ms})$. Ground switches then de-energise the link capacitance to a safe operational level via resistor discharge banks; this process may take tens of minutes depending on sizing of elements. It is assumed for these studies that the converter Station A circuit breaker fails to open in the event of a dc side fault leaving the dc link in an uncontrolled rectifying state [5]. The fault therefore requires to be cleared by an external means to avoid damage.

\section{Injection waveforms}

Voltage and current waveforms recorded at the distance relay during offline simulations will act as the input for the real time hardware-in-the-loop simulations. Three phase voltages and currents against a fixed time step $(100 \mu \mathrm{s})$ are produced from Simulink simulations and stored to a comma separated value (CSV) file for each fault scenario. These files are suitable for upload into the injection amplifier for hardware in the loop performance analysis.
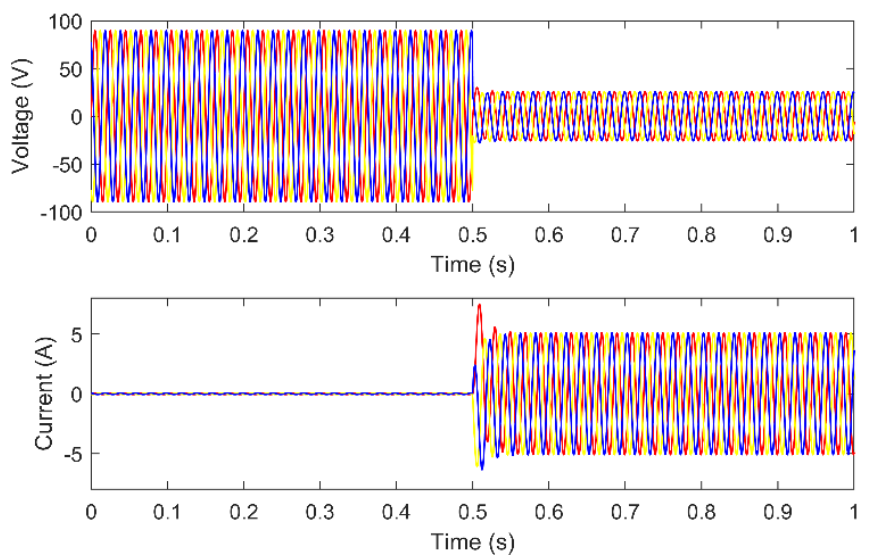

Fig. 5. Sample three phase injection current and voltage waveforms for a fault residing in $\mathrm{Z} 1$
A sample injection waveform showing both voltage and current is presented in Fig. 5. These values are secondary referred via a voltage transformer (VT) with ratio of $33,000 / 110 \mathrm{~V} / \mathrm{V}$ and a current transformer (CT) ratio of 1750/1 A/A. All faults were thrown at 0.5 seconds of simulation time, with the MVDC link exporting 5 MVA of power towards the external grid

\section{EXPERIMENTAL STUDIES}

Through the steps outline previously, several voltage and current profiles have been generated which are to be used as the input to the hardware in the loop laboratory experiment. The following sections describe how the protection IED, amplifier and controllers were configured. Results from the experiments are presented and discussed towards the end of this section.

\section{A. Protection Settings and Fault Location}

Protection settings for the distance relay are assigned in accordance with DNO protection guidance as discussed in Section II. The calculation for $\mathrm{Z} 4$ resistive and reactive line reaches are presented below using Equation (1) and (2). The line impedance angle is calculated via Equation (3). Note that the $\mathrm{Z4}$ line angle setting takes into account the converter reactor.

$$
\begin{gathered}
R_{Z 4} \approx 1.2 \times[(0.17 \times 5)+(0.3)+(0.17 \times 10)] \approx 3.42 \Omega \\
X_{Z 4} \approx 1.2 \times[(0.345 \times 5)+6.22+3.14+(0.345 \times 10)] \\
\theta=\tan ^{-1}\left(\frac{X_{\text {line }}}{R_{\text {line }}}\right)=\tan ^{-1}\left(\frac{0.3}{0.17}\right)=60.5^{\circ}
\end{gathered}
$$

Table II outlines setting parameters for the distance protection relay. Note that these values are provided in primary values and are not referred by means of the CT and VT ratios. Quadrilateral characteristics have been implemented for all zones due to limitations in the IEDs configuration software.

TABLE II

DISTANCE PROTECTION RELAY SETTING PARAMETERS

\begin{tabular}{ll}
\hline \hline \multicolumn{1}{c}{ Parameter } & \multicolumn{1}{c}{ Value } \\
\hline Line Angle $[Z 1-Z 3]\left(^{\circ}\right)$ & 60.5 \\
Line Angle $[Z 4]\left(^{\circ}\right)$ & 80.0 \\
Z1 Reach $(\Omega)$ & 1.374 \\
Z2 Reach $(\Omega)$ & 2.146 \\
Z3 Reach $(\Omega)$ & 6.010 \\
Z4 Reach $(\Omega)$ & 17.44 \\
Resistive Reach (All Zones) $(\Omega)$ & 3.420 \\
Z1 Fault Delay (phase \& earth) $(\mathrm{s})$ & 0.100 \\
Z2 Fault Delay (phase \& earth) $(\mathrm{s})$ & 0.500 \\
Z3 Fault Delay (phase \& earth) $(\mathrm{s})$ & 1.300 \\
Z4 Fault Delay (phase \& earth) $(\mathrm{s})$ & 0.200 \\
\hline \hline
\end{tabular}

\section{B. Hardware in the Loop Setup}

Fig. 6 displays a flow chart of the hardware in the loop setup. The control computer uploads waveforms into the programmable test amplifier via a parallel interface. The amplifier connects to the distance protection relay via shrouded test leads; three for voltage injections and three for current injections. The protection IED settings are configured 
via a setting file which is generated by the relay configuration software and uploaded via a serial interface.

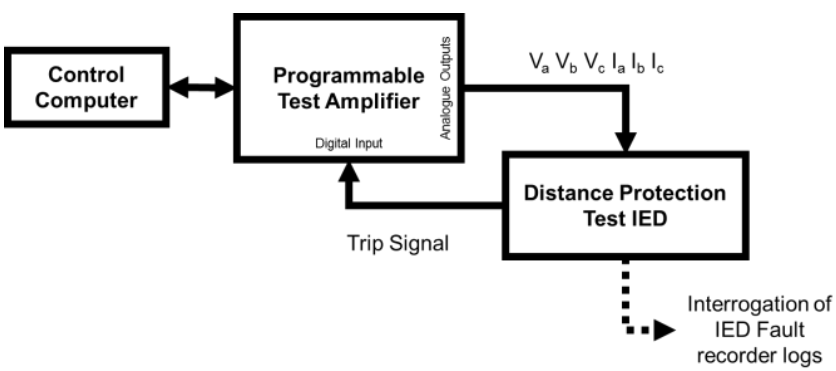

Fig. 6. Real time test setup diagram

The test experiment is initiated by the control computer. The simulation waveforms are injected into the relay. The tripping output contact of the relay connects back into the injection amplifier to allow trip times to be recorded. Further interrogation of events is achieved via the relay's human machine interface or by serial download. Fig. 7 and Fig. 8 display the experimental setup in the Dynamic Power Systems Laboratory at the University of Strathclyde.

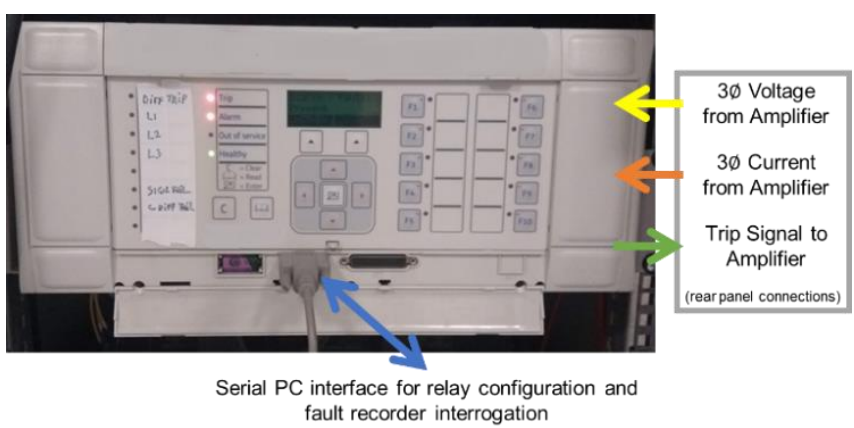

Fig. 7. Commercially available distance protection IED connecting to injection amplifier (Fig. 8)

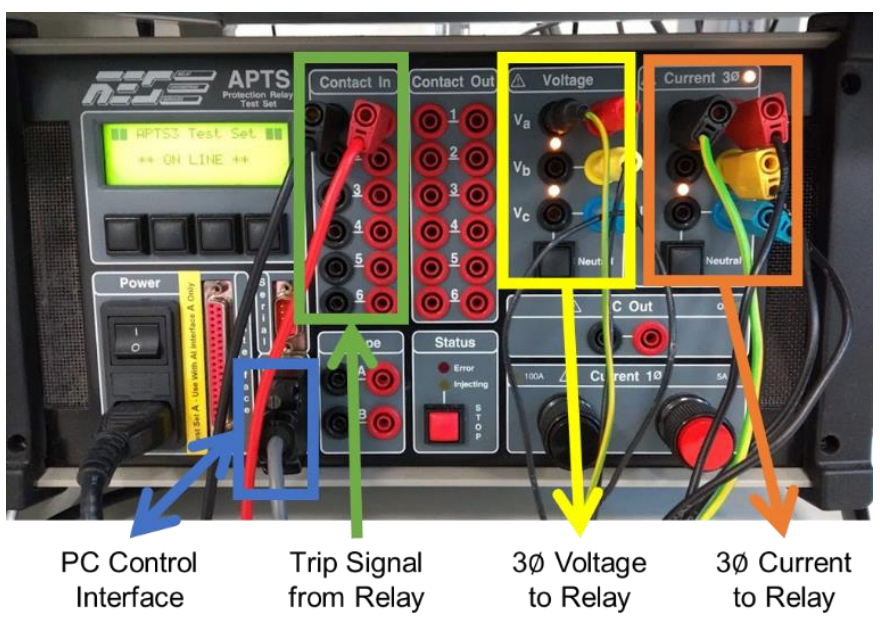

Fig. 8. Laboratory injection amplifier

\section{Experimental Results}

Four simulations are conducted for symmetrical faults in each of the protection zones as outlined previously. Each fault was thrown at $0.5 \mathrm{~s}$. The relay trip time and zone element are recorded in Table III for each of the four scenarios. Simplified R-X diagrams (only showing measurements between phase $\mathrm{A}$ and $\mathrm{B}$ ) for each simulation are presented in Fig. 9 - Fig. 12.

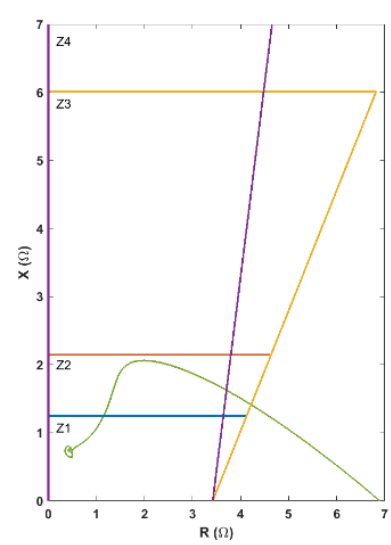

Fig. 9. R-X diagram for fault F1

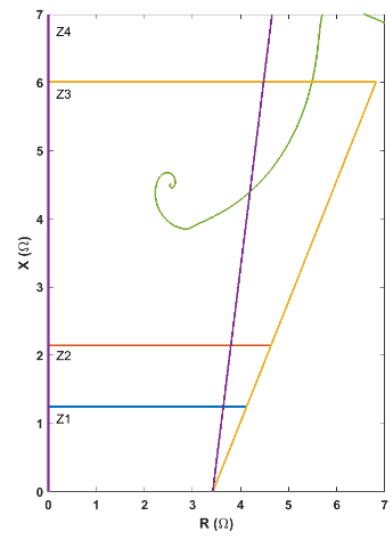

Fig. 11. R-X diagram for fault F3

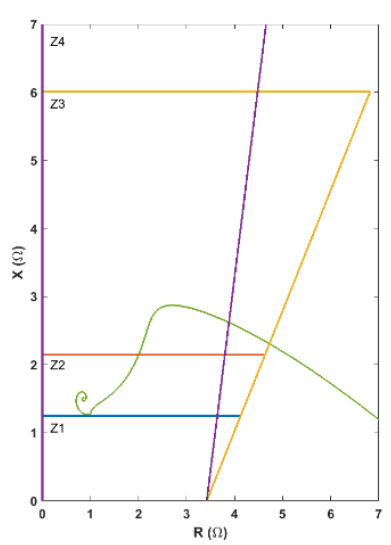

Fig. 10. R-X diagram for fault F2

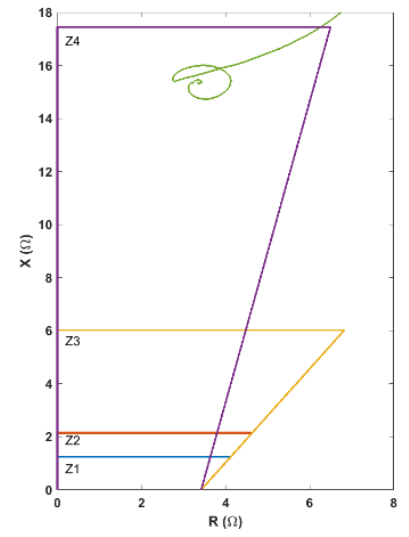

Fig. 12. R-X diagram for fault F4
TABLE III

RELAY LOG FOR INITIAL FAULT SIMULATIONS

\begin{tabular}{c|c|c|c|c|c}
\hline \hline \multirow{2}{*}{ Scenario } & \multicolumn{4}{|c|}{ Distance Zone Triggered } & Trip \\
& $\mathbf{Z 1}$ & $\mathbf{Z 2}$ & $\mathbf{Z 3}$ & $\mathbf{Z 4}$ & Time (s) \\
\hline F1 & $\bullet$ & $\bullet$ & $\bullet$ & $\bullet$ & 0.5212 \\
F2 & & $\bullet$ & $\bullet$ & $\bullet$ & 0.7351 \\
F3 & & & $\bullet$ & $\bullet$ & 0.7365 \\
F4 & & & & $\bullet$ & 0.7354 \\
\hline \hline
\end{tabular}

While the R-X diagram shows fault identification in the correct zone, due to the accelerated nature of the Z4 protection, faults in $\mathrm{Z} 2$ and $\mathrm{Z} 3$ are causing premature tripping. The standard logic of a distance protection relay assumes that the tripping time increases with increasing zone. Altering the relay tripping logic such to avoid accelerated tripping of $\mathrm{Z} 2$ and $\mathrm{Z} 3$ yielded experimental tripping times outlined in Table IV.

TABLE IV

RELAY LOG FOR CORRECTED FAULT SIMULATIONS

\begin{tabular}{c|c|c|c|c|c}
\hline \hline \multirow{2}{*}{ Scenario } & \multicolumn{3}{|c|}{ Distance Zone Triggered } & \multicolumn{2}{c}{ Trip } \\
& $\mathbf{Z 1}$ & $\mathbf{Z 2}$ & $\mathbf{Z 3}$ & $\mathbf{Z 4}$ & Time (s) \\
\hline F1 & $\bullet$ & $\bullet$ & $\bullet$ & $\bullet$ & 0.5206 \\
F2 & & $\bullet$ & $\bullet$ & $\bullet$ & 1.0166 \\
F3 & & & $\bullet$ & $\bullet$ & 1.8220 \\
F4 & & & & $\bullet$ & 0.7344 \\
\hline \hline
\end{tabular}

\section{Discussion}

From the real time experiments it has been observed that the commercially available distance protection IED can successfully respond to dc side faults in an accelerated manner while still being sensitive to ac system events in Z1, $\mathrm{Z} 2$ and $\mathrm{Z} 3$. However, the protection logic of relays requires 
alteration to avoid premature tripping for faults residing in $\mathrm{Z} 2$ and Z3. This modification is possible but additional setting parameters in the configuration software would make implementation simpler for end users. This relaying approach makes it possible to use existing distance protection IEDs in the future where MVDC technologies may be more prevalent. This method offers both backup functionality for MVDC primary protection but also offers cost savings to network operators as well as increased system confidence. Hardware in the loop simulations also show that it is possible to distinguish between ac and dc faults on parallel feeders.

\section{OVERVIEW OF MVDC ACTIVITY IN GREAT BRITAIN}

The work in this paper has been stimulated by growth observed in the medium voltage dc sector both within the UK and internationally. There are two notable demonstration projects currently under development and trial in the United Kingdom; funded via the Great Britain (GB) energy regulator OFGEM (Office of Gas and Electricity Markets). While both projects acknowledge MVDC technologies are not ready to be business as usual, they are keen to gain operational experience of the technology as they see dc being a major part of their future operation.

\section{A. Angle-DC}

SP Energy Networks, the DNO for central Scotland, Merseyside and North Wales, is currently installing an embedded MVDC link within a generation constrained region of its network [3]. The link is of 35 MVA power rating operating at $\pm 27 \mathrm{kV}$ and represents a $£ 14.8$ million innovation trial. The project has been triggered by a series of factors (including voltage constraints and a large renewable penetration in the area) which would otherwise require a more wide scale reinforcement or by limiting future connections. The link is additionally being used to prevent power from a new transmission connected nuclear plant flowing through the parallel $33 \mathrm{kV}$ distribution network.

The converter stations are based upon a cascaded three level neutral point clamped (NPC) arrangement derived from traction machine supplies. Each 'mini-converter' or module connects at $2.1 \mathrm{kV}$ ac which is derived from a multi-winding transformer. The dc voltage produced by each module is 4.5 $\mathrm{kV}$ with each having a power rating of $2.85 \mathrm{MVA}$ [13]. Fig. 13 presents a simplified electrical topology of an Angle-DC converter station.

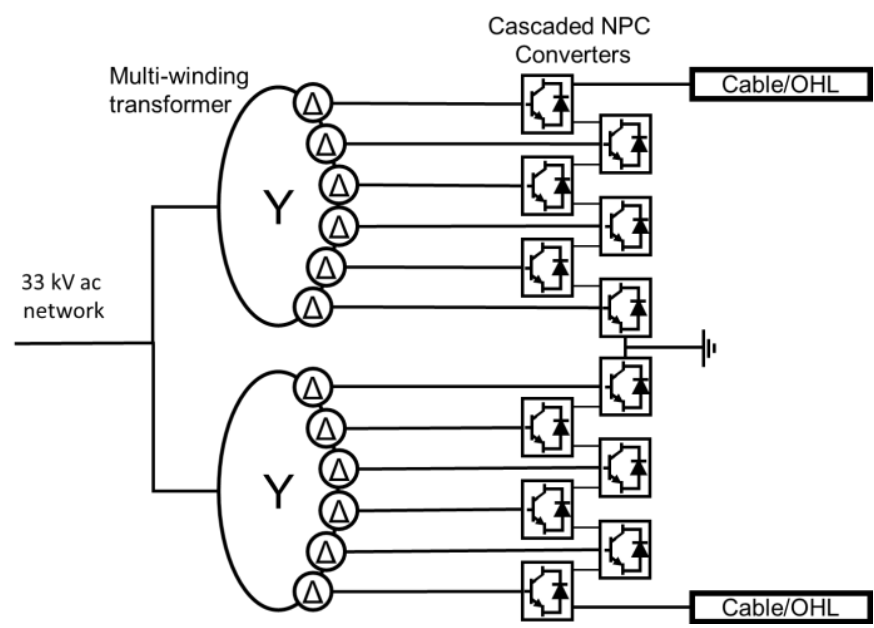

Fig. 13. Angle DC simplified station topology
The MVDC network is formed from two existing three wire ac circuits. Each circuit sees its three phases combined to form one pole of the dc link. The circuits are a mixture of overhead line (OHL) and cable sections. Each circuit takes a different geographical route and therefore means that a pole to pole fault is extremely unlikely.

SP Energy Network suggest that a $20 \%$ reduction in network losses expected from the successful project implementation will result in annual savings of approximately £630k [14]. The link is currently under construction and is scheduled to become operational in $\mathrm{mid} / \mathrm{late} 2019$.

\section{B. Network Equilibrium - Flexible Power Link}

While the Angle-DC project sees dc power transfer over multiple kilometres (through existing ac wayleaves), the Flexible Power Link (FPL), part of Western Power Distribution's (WPD) Network Equilibrium project has seen the installation of power electronic link within an existing distribution system substation [15]. WPD is the DNO responsible for the operation of distribution networks in Midlands, South West and Wales. The link consists of a 20 MVA / 5 MVAr capable back to back voltage source power electronic converter virtually connects two different $33 \mathrm{kV}$ distribution networks. Studies conducted by the DNO show that connection of these networks via conventional methods would cause issues such as circulating currents, protection grading difficulties and fault level constraints. The FPL work package was part of a wider project looking at improved voltage control within distribution networks which totalled $£ 13.1$ million.

The link is claimed to have the potential to unlock $36 \mathrm{MW}$ of latent grid capacity for DG connections via thermal and voltage management of the two virtually interconnected distribution network sectors. The technology involved is based upon flexible ac transmission system (FACTS) devices [16].

The FPL device is a containerised solution based upon existing static frequency converters (generally used for traction applications which allows for offsite construction and therefore reduced installation time in the substation environment. This approach also allows the FPL device to be relocated more readily should network conditions change.

\section{FURTHER WORK AND DISCUSSION}

There have been a number of future work opportunities identified for this research topic which build on the work conducted to date. Suitable time grading margins need to be defined between ac distance protection and converter protection for dc side faults. A wider range of system event analysis is required to ensure security of the protection system across multiple operation scenarios. This could include remote transformer energisation. Converter fault ride through characteristics for ac side faults has been shown to potentially effect clearance times of distance protection devices for both dc links and embedded generation [6].

While these simulations were performed on a commercially available distance protection relay, it is important that injection tests are conducted on a larger cross-section of 
available IEDs to prove applicability across relay manufacturers and device age.

This research may be transferable to transmission connected high voltage dc (HVDC) schemes however it is noted that the higher $\mathrm{X} / \mathrm{R}$ ratio of a transmission system may decrease the stability of the proposed solution.

While these studies have been presented for a two level symmetrical monopole arrangement, it is hypothesised that this approach would be applicable across other dc conversion topologies given the similar fault current paths through such devices. It is acknowledged that fault blocking converter such as full bridge multi-level modular converters (MMC) would not benefit from such a solution. It could be argued that providing dependable protection solutions (both main and backup) for simpler converter topologies, rather than more advanced topologies, may increase uptake of embedded MVDC in distribution systems due to reduced station costs and complexity.

\section{CONCLUSIONS}

Remote backup protection of an MVDC link is likely to give grid operators increased confidence when deploying $\mathrm{dc}$ in their networks. Backup protection which is provided via existing operational protection IEDs offers an elegant solution ultimately leading to cost savings for future MVDC schemes. This paper suggests what setting parameters need to be selected on a digital distance protection relay in order to make the device sensitive to remote dc side faults. These recommendations have been proven under a real time hardware in loop simulations. It is important that tripping logic is correctly defined such that IEDs do not trip too quickly for faults which reside in both the Z4 quadrilateral and lower zones. Future work packages have been identified.

\section{ACKNOWLEDGMENT}

This work has been supported through the Engineering and Physical Sciences Research Council (EPSRC) Centre for Doctoral Training in Future Power Networks and Smart Grids (EP/L015471/1).

\section{REFERENCES}

[1] L. Hunter, C. Booth, S. Finney and A. J. Ferré, "MVDC Network Balancing for Increased Penetration of Low Carbon Technologies," in IEEE PES Innovative Smart Grid Technologies Conference Europe (ISGT-Europe), Sarajevo, Bosnia-Herzegovina, 2018.

[2] F. Mura and R. W. De Doncker, "Preparation of a Medium-Voltage DC Grid Medium-Voltage DC Grid," Aachen, 2012.

[3] SP Energy Networks, “Angle DC," [Online]. Available:

https://www.spenergynetworks.co.uk/pages/angle_dc. aspx. [Accessed December 2016].
[4] L. Hunter, C. Booth, A. J. Ferre and S. Finney, "MVDC for enhanced utility scale distribution power delivery and control," in 52nd International Universities Power Engineering Conference (UPEC), Heraklion, 2017.

[5] F. P. Page, Analysis in Circuit Breaker Performance Requirements for High-Voltage DC Networks, 2016.

[6] L. Hunter, C. Booth, A. Dysko, S. Finney and A. Junyent-Ferré, "The impact of MVDC upon conventional distance protection schemes in hybrid acdc distribution networks," in IET ACDC 2019, The 15th IET International Conference on AC and DC Power Transmission (ACDC 2019), 2019.

[7] GE Power Management, "Distance Relays Fundamentals," April 2002. [Online]. Available: https://store.gegridsolutions.com/faq/documents/alps/ GER-3966.pdf. [Accessed March 2018].

[8] J. D. Glover, M. S. Sarma and T. J. Overbye, "Line Protection with Impedance (Distance) Relays," in Power System - Analysis and Design, Stamford, CT, USA, Cengage Learning, 2008, pp. 551-557.

[9] Alstom Grid, Network Protection \& Automation Guide, 2011.

[10] SSEN, "Long Term Development Statement," Scottish Hydro Electric Power Distribution, November 2016. [Online]. [Accessed January 2017].

[11] A. Egea-Alvarez, A. Junyent-Ferré and O. GomisBellmunt, "Active and reactive power control ofgrid connected distributed generation systems," in Modeling and Control of Sustainable Power Systems, Berlin, Springer, 2012, pp. 47-81.

[12] DCODE, "The Distribution Code for licensed DNOs of Great Britain - Issue 29," February 2018.

[13] A. Mooon and K. Smith, "Developments in the AngleDC project; Project challenges, developments and findings to date (SP Energy Networks)," in ECPE Workshop - DC Grids, Technologies and Applications, Aachen, Germany, 2018.

[14] SP Energy Networks, "Electricity NIC submission: SP Energy Networks - ANGLE-DC," 30 November 2015. [Online]. Available: https://www.ofgem.gov.uk/sites/default/files/docs/ang le_submission.pdf. [Accessed 14 January 2019].

[15] Western Power Distribution, "Network Equilibrium," 2015-2019. [Online]. Available: https://www.westernpower.co.uk/projects/networkequilibrium. [Accessed 12 October 2018].

[16] Western Power Distribution, "Low Carbon Networks Fund submission from Western Power Distribution Network Equilibrium," 24 November 2014. [Online]. Available: https://www.westernpower.co.uk/downloads/2677. [Accessed 18 January 2019]. 